\title{
Combustion of Fuel JP8-1: Mechanism and Reaction Kinetics Based on ReaxFF MD
}

Yang Liu ${ }^{a, b}$, Xin Wei ${ }^{a}$, Weizhen Sun ${ }^{a,}$, and Ling Zhao ${ }^{a, b}$

a State Key Laboratory of Chemical Engineering, School of Chemical Engineering,

East China University of Science and Technology, Shanghai 200237, China

b School of Chemistry \& Chemical Engineering, XinJiang University, Urumqi 830046, China

\section{S1. Calculation Program of the Reaction Rate Constant}

(1). Open a terminal:

- on Linux: Ctrl+Alt+T usually works, otherwise browse your application menus

- on Windows: double click adf_command_line.bat in your ADF installation directory (e.g. C:/ADF2016.106), enter 'sh' to go to a shell

(2). cd into the directory where your trajectories are located with the terminal

(3). Running ChemTraYzer:

assuming your trajectory is called traj.rxkf

a) run the processing step (with default values)

\$ADFBIN/startpython \$ADFHOME/scripting/chemtrayzer/processing_scm.py traj.rxkf 
b) run the analysis step (with default values)

\$ADFBIN/startpython \$ADFHOME/scripting/chemtrayzer/analyzing_scm.py traj.rxkf

you will probably want to adjust the settings. The available options are shown by calling the scripts without arguments, e.g.

\$ADFBIN/startpython \$ADFHOME/scripting/chemtrayzer/analyzing_scm.py

(4). Results

there are several output files, the most relevant ones being:

reac.spec.tab

1st line: indices of the species, e.g. S1;S2;S3;etc...

2nd line: SMILES of the species, e.g. ;C;CC;CCO;CCOO;CO;O;[H];etc...

3rd - end: timestep, counts of the species, e.g. $150 ; 0 ; 0 ; 2 ; \ldots / /$ step 150 , current count of $\mathrm{S} 3==3$

reac.rate.tab

1st line: indices of the reactions, e.g. $\mathrm{t}$ [steps];R0;R0*;R1;R1*;// a "*" marks the back reaction

2nd line: the reactions formulated with the species indices, e.g. ; $\mathrm{S} 0+\mathrm{S} 0+\mathrm{S} 0+\mathrm{S} 13$ -> S65; // see reac.spec.tab

3rd line: the reactions formulated with sum formulas, e.g. $\mathrm{CH} 3+\mathrm{H}->\mathrm{CH} 4$, etc...

4th -...: timestep;rate constant of the reaction; rate constant of the back reaction; etc... 
reac.pic/

folder containing the 2D structures (if obabel was able to create them)

reac.pic/xyz/ xyz files of the species as they first appeared in the trajectory 